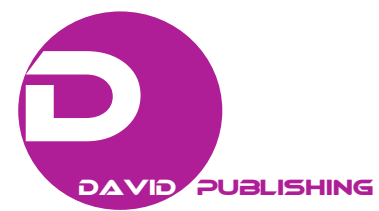

\title{
Midgut Volvulus: Acute Abdomen in an Adult Patient
}

\author{
Hamri Asma, Rabbani Khalid, Louzi Abdelouahed and Finech Benasser \\ Department of Surgery, Mohammed the Sixth University Hospital, Marrakech 40000, Morocco
}

\begin{abstract}
Midgut malrotation is an anomaly of intestinal rotation which occurs during fetal development and usually presents in the neonatal period. It is rare for malrotation to present in adulthood. The main purpose of this paper is to show how the clinical picture may be expected to vary and to emphasize the importance of "shock" in massivevolvulus. We present a case of midgut volvulus in an adult male patient (60 year old) who presented with cramping generalized right abdominal pain and vomiting of two days duration. An abdominal ultrasound showed peritoneal effusion and greek distension. An emergency laparotomy showed typical Ladd's bands and small bowel distension. All the intestine from the duodenum to the left colic flexure was found to be suspended on a single dorsal mesentery. This mesentery arose from a narrow base high up in the abdomen and reached down to the pelvic brim. There were numerous areas of fine shiny fibrosis on the small bowel corresponding to sites of previous constriction when a midgut volvulus occurred. A detorsion of the twisted mesentery, lysis of the bands, appendectomy were performed. Complete resolution of symptoms is noted. Acute volvulus of the small intestine is one of the most serious abdominal emergencies. Diagnosis may be difficult and delay in operative intervention can be disastrous.
\end{abstract}

Key words: Midgut malrotations, adult patient.

\section{Background}

Midgut volvulus is a life-threatening complication of small bowel malrotation [1]. It usually occurs early in life and is a very rare finding in adults. It is however the most common cause of bowel obstruction in the adult with malrotation [2], we present a new case of a midgut volvulus in adult patient.

\section{Case Report}

A 60 year-old man, who presented with cramping generalized right abdominal pain and vomiting of two days duration. An abdominal ultrasound showed peritoneal effusion and greek distension. An emergency laparotomy showed typical Ladd's bands and small bowel distension. All the intestine from the duodenum to the left colic flexure was found to be suspended on a single dorsal mesentery. The caecum was positioned in the mesogastric region. This

Corresponding author: Finech Benasser, Ph.D., research field: surgery. mesentery arose from a narrow base high up in the abdomen and reached down to the pelvic brim. There were numerous areas of fine shiny fibrosis on the small bowel corresponding to sites of previous constriction when a midgut volvulus occurred (Fig. 1). A detorsion of the twisted mesentery, lysis of the bands, appendectomy were performed. Complete resolution of symptoms is noted.

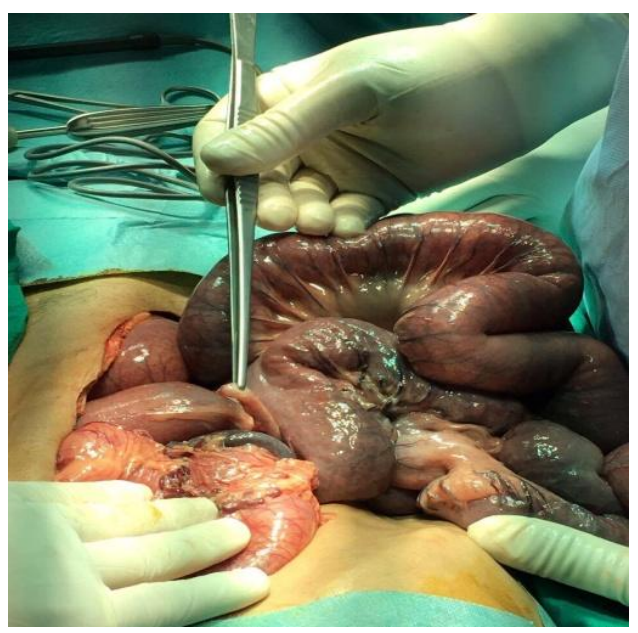

Fig. 1 Twisting of the small bowel. 


\section{Discussion}

Though midgut malrotation is a common cause of intestinal obstruction in the newborn, scant attention is given to this developmental anomaly as a cause of symptoms in adults [1].

In malrotation, the mesenteric root which, in normal conditions, forms a diagonal line from the Treitz's ligament to the caecum, is shortened. The ligament of Treitz is located in these cases more inferiorly and to the right of its normal position [2]. This short mesenteric attachment of the small bowel can permit rotation of the small bowel around the axis of the SMA, thereby resulting in midgut volvulus [3]. Clinical findings depend on the severity of the twist around the SMA and include abdominal pain, bilious vomiting, and bloody stools. If twisting exceeds 3.5 turns, ischemia of the bowel will occur thereby resulting in necrosis of the entire jejunum level than the femoral ring [4]. Inversion of the SMA and SMV on US is very suggestive of intestinal malrotation but not totally specific for its diagnosis. Signs as duodenal obstruction at the distal part of the duodenum, a solitary and hyperdynamic pulsating SMA, and thick-walled loops of bowel below the duodenum and to the right of the spine in association with peritoneal fluid have been reported before but are rather subjective and not specific enough for diagnosing midgut volvulus [5].

\section{Conclusion}

Malrotation leading to midgut volvulus is rare in adults. The CT findings in midgut volvulus can be pathognomonic if the classic whirl pattern around a central superior mesentenc artery is seen. CT allows the rapid diagnosis and surgical treatment of this unusual condition in the adult patient presenting with abdominal pain or bowel obstruction of uncertain cause.

\section{References}

[1] Akile, Z., Bercis, İ. U., Şükrü, A. D., Zülfü, B., Sezgin, Z., Faik, Y., Bekir, Ş., and Nilüfer, A. B. 2016. "Adult Midgut Malrotation Presented with Acute Bowel Obstruction and Ischemia." International Journal of Surgery Case Reports 22: $5-7$.

[2] Husberg, B., Salehi, K., Peters, T., Gunnarsson, U., Michanek, M., Nordenskjöld, A., and Strigård, K. 2016. "Congenital Intestinal Malrotation in Adolescent and Adult Patients: A 12-Year Clinical and Radiological Survey." Springerplus 5: 245.

[3] Bernstein, S. M., and Russ, P. D. 1998. "Midgut Volvulus: A Rare Cause of Acute Abdomen in an Adult Patient." Am. J. Roentgenol. 171 (3): 639-41.

[4] Sahu, S. K., Raghuvanshi, S., Sinha, A., and Sachan, P. K. 2012. "Adult Intestinal Malrotation Presenting as Midgut Volvulus: Case Report." India. Cer. San. D. (J. Surg. Arts) 5 (1): 18-21. 